\title{
Electron pockets and pseudogap Dirac point in underdoped cuprate superconductors
}

\author{
K.A.G. Fisher ${ }^{1,2}$, E.J. NiCOL ${ }^{1,2}$ and J.P. CARBOtTE ${ }^{3,4}$ \\ Department of Physics, University of Guelph, Guelph, Ontario, Canada N1G 2W1 \\ Guelph-Waterloo Physics Institute, University of Guelph, Guelph, Ontario, Canada N1G 2W1 \\ Department of Physics and Astronomy, McMaster University, Hamilton, Ontario, Canada L8S $4 M 1$ \\ The Canadian Institute for Advanced Research, Toronto, Ontario, Canada M5G $1 Z 8$
}

\author{
PACS 74.72.-h - Cuprate superconductors \\ PACS 74.72.Kf - Pseudogap regime \\ PACS 74.20.Mn - Nonconventional mechanisms (spin fluctuations, polarons and bipolarons, res- \\ onating valence bond model, anyon mechanism, marginal Fermi liquid, Lut- \\ tinger liquid, etc.)
}

\begin{abstract}
We consider a model of the pseudogap specifically designed to describe the underdoped cuprates and which exhibits particle-hole asymmetry. The presence of electron pockets, besides the usual hole pockets, leads to the appearance of new vectors beyond the usual so-called octet model in the joint density of states (JDOS), which underlies the analysis of Fourier-transform scanning tunneling spectroscopy (FT-STS) data. These new vectors are associated with distinct patterns of large amplitude in the JDOS and are expected to occur primarily at positive bias. Likewise a pseudogap Dirac point occurs at positive bias and this point can be determined either through FT-STS or through extrapolation of data from the autocorrelation function of angleresolved photoemission spectroscopy.
\end{abstract}

Prominent in the underdoped cuprate superconductors is the existence of a pseudogap in the excitation spectrum which opens above $T_{c}$ but below a temperature $T^{*}$. 1 Whether this gap is the same as the superconducting energy gap or is a manifestation of a competing order independent of the superconductivity remains an open and central question. Indeed, it is not clear that the two en- ergy scales seen in experiments [2 4] arise from two sepárate gaps [5]. If there are two distinct gaps of $d$-wave symmetry, they each will exhibit a Dirac point, a point at which linear dispersions cross, at a different energy and momentum in the band structure. Evidence for a single gap would favour theoretical proposals of preformed Cooper pairs above $T_{c}$ which phase lock at this temperature. 6] On the other hand, models with competing order typically have a second energy gap scale and some form of Fermi surface reconstruction which gives rise to the possibility of hole and electron pockets. [7, 8, While hole pockets or arcs have been seen in ARPES experiments, 911 recently the observation of electron pockets from quantum oscillations in high magnetic fields has been reported. 12 Furthermore, the preformed pair theory gives rise to particle-hole symmetric quantities whereas competing orders can exhibit particle-hole asymmetry. [13, 14] As two-gap scenarios typically arise from strong correlations due to the nearby presence of the antiferromagnetic Mott insulating state shown in the phase diagram of fig. 1(a), the AFM Brillouin zone boundary plays a significant role in determining the reformation of the large Fermi surface to small pockets with underdoping as shown in fig. 1)(b). Given that the underlying mechanism of superconductivity is most likely to be attributed to antiferromagnetic spin fluctuations, as directly evidenced by experiment [15, both the pseudogap and superconducting gap are expected to reflect the same symmetry which is known to be $d$-wave in the cuprates [16. Thus, the gaps will exhibit Dirac points in the $(\pi, \pi)$ direction with that for superconductivity on the Fermi surface (taken as $\omega=0$ in energy) and that for the pseudogap shifted to positive energy and at a different $\boldsymbol{k}$ point in the Brillouin zone [14]. In this letter, we demonstrate that the existence of electron pockets will lead to additional characteristic features in the quasiparticle interference (QPI) patterns [17,18] obtained from Fourier transform scanning tunneling spectroscopy (FT- 
a

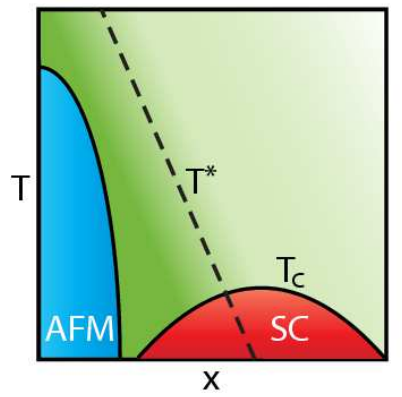

c

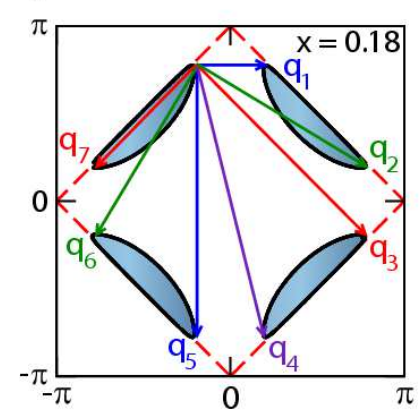

b

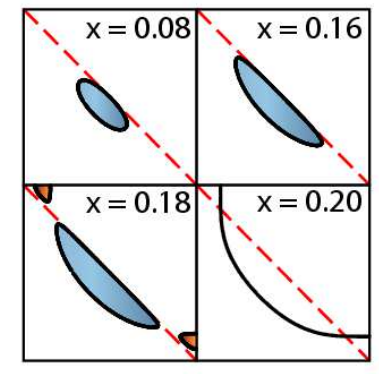

d

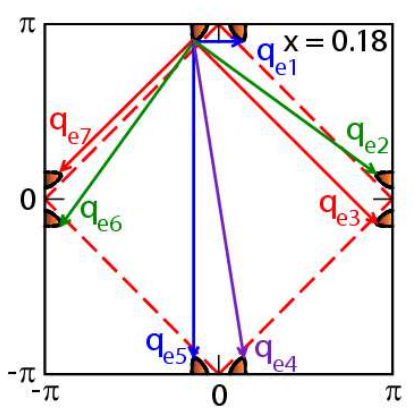

Fig. 1: (Colour online) (a) Generic phase diagram of the holedoped cuprates showing the antiferromagnetic Mott insulator (AFM) region, the superconducting dome (SC) and the $T^{*}$ line indicating the onset of the pseudogap phase at lower doping $x$ and temperature T. (b) The reconstruction of the Fermi surface in the first quadrant of the square Brillouin zone as the doping is varied from underdoped $(x=0.08,0.16,0.18)$ to optimally doped $(x=0.2)$. When no pseudogap is present, as in the case of $x=0.2$ which is taken as the quantum critical point where the $T^{*}$ line goes to zero, then there is a large Fermi surface. With slight underdoping electron pockets (red) and a hole pocket (blue) appear about the AFM Brillouin zone boundary (red dashed line). These pockets shrink with underdoping. (c) The octet model $\boldsymbol{q}$ 's of AC-ARPES and FT-STS which connect points of high density of states associated with the hole pockets (or arcs in some models). (d) The electron pockets for $x=0.18$ will also be connected by eight $\boldsymbol{q}_{e}$ 's. Not shown here are possible interband $\boldsymbol{q}$ 's which are in addition to the intraband $\boldsymbol{q}$ 's shown in (c) and (d).

STS). Furthermore, we discuss the various signatures of a particle-hole asymmetric pseudogap that may lead to the possible identification of the pseudogap Dirac point through FT-STS or via the extrapolation of information from the autocorrelation function of angle-resolved photoemission spectroscopy (AC-ARPES) [19,20].

It was shown [19] that there is a direct correspondence between the QPI measured in FT-STS and the ACARPES. This important work confirmed that an evaluation of the joint density of states (JDOS) in terms of the spectral function $A(\boldsymbol{k}, \omega)$ :

$$
J \operatorname{DOS}(\boldsymbol{q}, \omega)=\frac{1}{V} \sum_{\boldsymbol{k}} A(\boldsymbol{k}, \omega) A(\boldsymbol{k}+\boldsymbol{q}, \omega)
$$

will give rise at fixed energy $\omega$ to a series of strong peaks

at the tips of eight $\boldsymbol{q}$ vectors (one of which is 0 ) which is known as the octet model 21. For a quasiparticle dispersion given by $E_{\boldsymbol{k}}$, these $\boldsymbol{q}$ 's map a point of high density of states due to $1 / \nabla E_{\boldsymbol{k}}$ to another such point as shown in fig. 1(c). The patterns for the JDOS formed experimentally by an autocorrelation function of the ARPES $A(\boldsymbol{k}, \omega)$ agree well with the QPI found in FT-STS, which is based on the same physics. [19] We now show from the JDOS that AC-ARPES, indirectly, and the QPI FT-STS, more directly, can provide a unique way for determining both the energy and momentum information associated with the pseudogap Dirac point and also electron pockets.

To provide a concrete demonstration of the expected signatures of a second Dirac point, electron pockets and particle-hole asymmetry, we adopt the model of Yang, Rice and Zhang 22] who provide a phenomenological ansatz for a Green's function which represents a pseudogap state in the cuprates. This model has been developed out of prior work on t-J models, Hubbard ladders and resonating valence bond spin liquid theory [22] and has proven to be quite effective at describing a large body of experimental data [1, 14, 22, 35. In this model, the coherent part of the Green's function in the pseudogap state has the form of

$$
G(\boldsymbol{k}, \omega)=\frac{g_{t}}{\omega-\epsilon(\boldsymbol{k})-\Sigma_{\mathrm{pg}}(\boldsymbol{k}, \omega)},
$$

where the electron self-energy is given by $\Sigma_{\mathrm{pg}}(\boldsymbol{k}, \omega)=$ $\left|\Delta_{\mathrm{pg}}(\boldsymbol{k})\right|^{2} /\left[\omega+\epsilon_{0}(\boldsymbol{k})\right]$. Here, $g_{t}=2 x /(1+x)$ is a Gutzwiller factor that reflects strong correlations and a reduction in coherence with the approach toward the AFM Mott insulator. The pseudogap is taken to be $\Delta_{\mathrm{pg}}(\boldsymbol{k})=\Delta_{\mathrm{pg}}(x)\left[\cos \left(k_{x} a\right)-\cos \left(k_{y} a\right)\right] / 2$, with $\Delta_{\mathrm{pg}}(x)=$ $0.6(1-x / 0.2)$. The band structure $\epsilon(\boldsymbol{k})$ is taken to be a third nearest-neighbour tight-binding dispersion and $\epsilon_{0}(\boldsymbol{k})=-2 t_{0}\left[\cos \left(k_{x} a\right)+\cos \left(k_{y} a\right)\right]$, where $\epsilon_{0}(\boldsymbol{k})=0$ gives the AFM Brillouin zone boundary shown in fig. 1. Indeed, it is the fact that $\epsilon_{0}(\boldsymbol{k})$ appears in the electron self-energy $\Sigma_{\mathrm{pg}}(\boldsymbol{k}, \omega)$, instead of $\epsilon(\boldsymbol{k})$, that the pseudogap opens away from the Fermi surface $\epsilon(\boldsymbol{k})=0$ on a surface defined by $\epsilon(\boldsymbol{k})+\epsilon_{0}(\boldsymbol{k})=0$, giving rise to particle-hole asymmetry in this model. Further modifications of this form for a superconducting gap $\Delta_{\mathrm{sc}}(\boldsymbol{k})=\Delta_{\mathrm{sc}}(x)\left[\cos \left(k_{x} a\right)-\cos \left(k_{y} a\right)\right] / 2$, where $\Delta_{\mathrm{sc}}(x)=0.14\left[1-82.6(x-0.2)^{2}\right]$, are standard and given in refs. [14, 25], where the typical band structure parameters used here may also be found. (Note, that prior work on the JDOS has been done in this model [31, however, a theory was used for the superconducting state which has since been revised 25] and so results of ref. 31] differ from ours in the presence of superconductivity.) While we use a particular model for illustration, other models with particle-hole asymmetry would also give qualitatively similar results. The pockets of fig. 1(b) are a result of this model and it should be noted that at the Fermi energy the AFM Brillouin zone side has small quasiparticle weight and hence these pockets can appear as arcs in experiment. Experimental evidence for these types of 


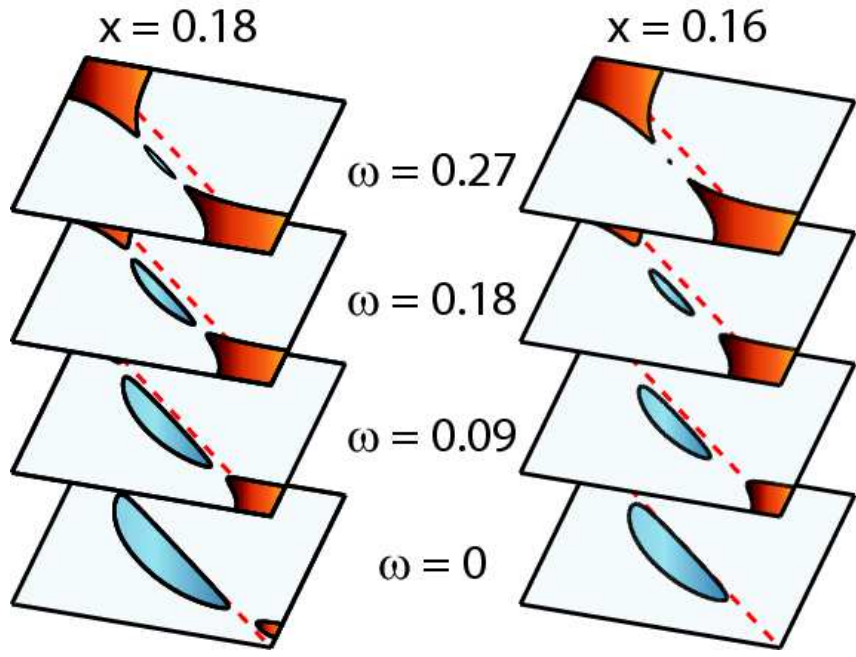

Fig. 2: (Colour online) Illustration of the evolution of hole and electron pockets for positive energy cuts of the energy bands. For $x=0.18$, electron pockets exist at $\omega=0$ and grow as the hole pocket shrinks. The $x=0.16$ case demonstrates that even if electron pockets do not exist at $\omega=0$, they will appear at higher positive bias. For $x=0.16$, at the largest energy shown, i.e. $\omega=0.27$ in units of $t_{0}$, the hole pocket has shrunk to a point which is the pseudogap Dirac point. For $x=0.18$, this will be reached at greater energy.

pockets in agreement with this theory has been recently presented in ref. [1]. Figure 1(d) illustrates that should electron pockets exist, they will also give rise to new peaks in the JDOS which would be seen in experiment. Note that while it might appear that only a narrow range of doping gives rise to electron pockets, as seen in fig. 1(b), it is shown in fig. 2 that electron pockets will appear at finite bias on the positive side even when not present at $\omega=0$. Moreover, due to the formation of Bogoliubov quasiparticles in the superconducting state, an image of these pockets can appear at negative bias. 14, 27 The pseudogap Dirac point is seen in this figure as the single point in the top right hand sheet for $x=0.16$. It will be found at positive energy along the nodal direction about halfway in momentum between the Fermi surface at $\omega=0$ and the AFM Brillouin zone. 14 The observation of electron pockets and the pseudogap Dirac point at positive bias poses a problem for ARPES which sees only the occupied states at negative energies, however, ARPES can potentially be used to obtain very useful but indirect information on the Dirac point from the particle-hole asymmetry.

Clearly, with the Dirac point at positive energy, an asymmetry exists in this situation. To discuss this point, we show in fig. 3 the particle-hole asymmetry that exists in the JDOS. While our prior figures were solely about the pseudogap state, this figure now includes superconductivity. In the pure pseudogap state, particle-hole asymmetry would occur at all biases but the presence of superconductivity restores the symmetry for energies below the
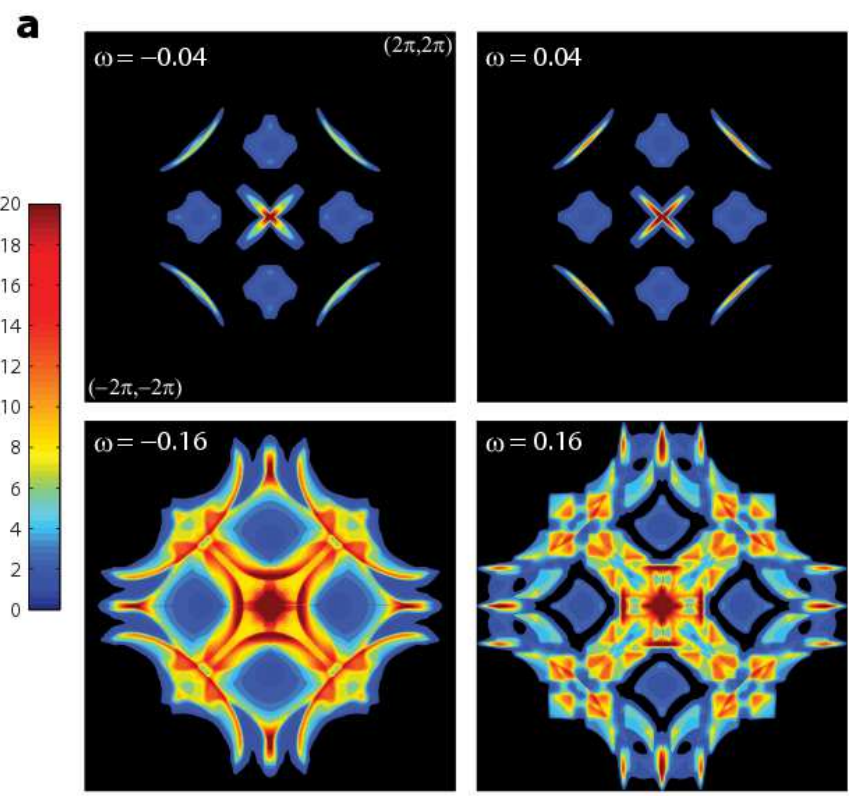

b

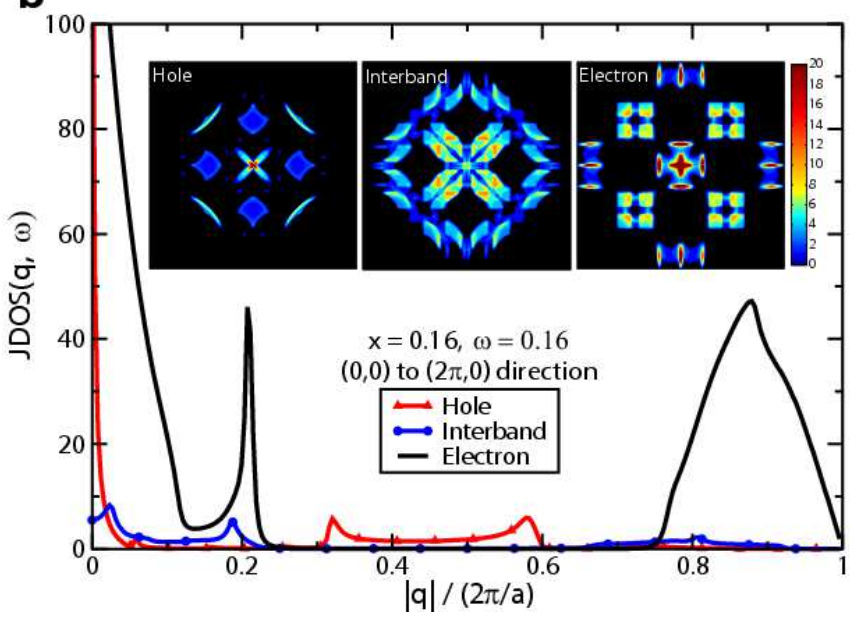

Fig. 3: (Colour online) (a) The JDOS in the superconducting state with the pseudogap present for $x=0.16$. Each colour map is shown for $\boldsymbol{q}$ varying from $(-2 \pi,-2 \pi)$ in the lower left corner to $(2 \pi, 2 \pi)$ in the upper right corner of the square. The left (right) frames are for negative (positive) bias. The top frames are for $|\omega|=0.04 t_{0}$ which is below the superconducting gap maximum $\Delta_{\mathrm{sc}}=0.12 t_{0}$. The bottom two frames are for $|\omega|=0.16 t_{0}$, well above $\Delta_{\mathrm{sc}}$. (b) The JDOS in the $(0,0)$ to $(2 \pi, 0)$ direction showing intraband (hole and electron) and interband contributions to the autocorrelation function at energy $\omega=0.16 t_{0}$. The inset shows the JDOS in all directions of $\boldsymbol{q}$ decomposed into the three components.

relevant superconducting energy scale. This is seen in fig. 3(a) where the top frames show the JDOS at positive and negative biases and the energy $|\omega|<\Delta_{\mathrm{sc}}$. These two frames show the particle-hole symmetry imposed by superconductivity. The bottom two frames for $|\omega|>\Delta_{\mathrm{sc}}$ illustrate the particle-hole asymmetry due to the pseudogap state. Using the case of $\omega=0.16 t_{0}$, fig. 3 (b) shows a 
decomposition of the colour map into its contributions for the hole intraband, electron-hole interband, and electron intraband pieces, respectively (see inset)and the strength of the JDOS for a cut along the center for $\boldsymbol{q}=(0,0)$ to $(2 \pi, 0)$. For this direction, the electron JDOS contribution dominates and the interband component is not important. Note that the JDOS shown here should be scaled by a factor of $g_{t}^{2} / 4 \pi^{2}$ in the model used here. The inset colour maps confirm the hole JDOS to be as shown before in prior works but in addition illustrate a feathered pattern for the interband JDOS and a distinctive 4-square pattern for the electron JDOS which also dominates the full $\boldsymbol{q}$-map in the lower right frame of (a). Indeed, one sees that there are very different characteristic fingerprints of electron pockets versus hole or interband and while much attention has been paid to the hole pockets, these other features can be distinguished in the composite colour map for $\omega=0.16$ in fig. 3(a). Here, as we will focus on the JDOS for $\boldsymbol{q}$ varying from $(0,0)$ to $(2 \pi, 0)$ only for brevity, we emphasize that the potential interband JDOS peaks are small in this direction and our discussion can focus solely on the independent electron and hole features.

Figure 4 emphasizes the typical JDOS curves and $\mathbf{q}$ dispersions for the case of no gaps, with a pseudogap and for both gaps present. Particle-hole asymmetry is imposed by the pseudogap and electron pockets give rise to new $\boldsymbol{q}$ 's at positive bias. The pseudogap-only case was obtained previously in ref. 31 for the negative bias range of $\omega=-0.1 t_{0}$ to 0 and our results appear to be in agreement in that range. These authors refer to this behaviour as non-dispersive but we find it to be dispersive on our expanded scale. With superconductivity, the $\boldsymbol{q}_{\mathbf{5}}$ and $\boldsymbol{q}_{\mathbf{1}}$ form an X-structure in the $\boldsymbol{q}$ versus $\omega$ plot with crossing point at $\omega=0$. This is the superconducting Dirac point and it occurs at a specific value of $|\boldsymbol{q}|$ which identifies the Fermi momentum $k_{F}=|\boldsymbol{q}| / \sqrt{2}$ in the nodal direction. The energy onset of the $\mathrm{X}$ is set by the maximum of the superconducting gap on the hole pocket while the onset of the electron $\boldsymbol{q}_{\boldsymbol{e} \mathbf{1}}$ and $\boldsymbol{q}_{\boldsymbol{e} \mathbf{5}}$ approximately corresponds to the pseudogap energy and is more rigorously identified as an energy scale $\Delta_{\mathrm{pg}}^{+}$discussed in ref. [14. Note that in fig. $4(\mathrm{e})$, the $\boldsymbol{q}_{\mathbf{1}}$ and $\boldsymbol{q}_{\mathbf{5}}$ merge towards a point at positive bias and in spite of the distortion due to superconductivity in fig. 4(f), the same overall trend in $\boldsymbol{q}_{\mathbf{1}}$ and $\boldsymbol{q}_{\mathbf{5}}$ is maintained. This point of merging is the pseudogap Dirac point. As a final comment, the superconducting and pseudogap Dirac point occur at mid range values of $\boldsymbol{q}$ concurrent with the nodal direction, whereas the electron pocket $\boldsymbol{q}$ 's are either large or small in magnitude as expected for features in the JDOS which arise from the near antinodal direction.

Figure 5 emphasizes the pseudogap Dirac point more clearly where for stronger underdoping the Dirac point shifts to smaller energy as tracked by the black arrows in frames (a)-(c). Likewise, this point is at a different $|\boldsymbol{q}|$ than the superconducting Dirac point. With nearest neighbour hopping $t_{0}$ taken to be $150-350 \mathrm{meV}$ in fitting
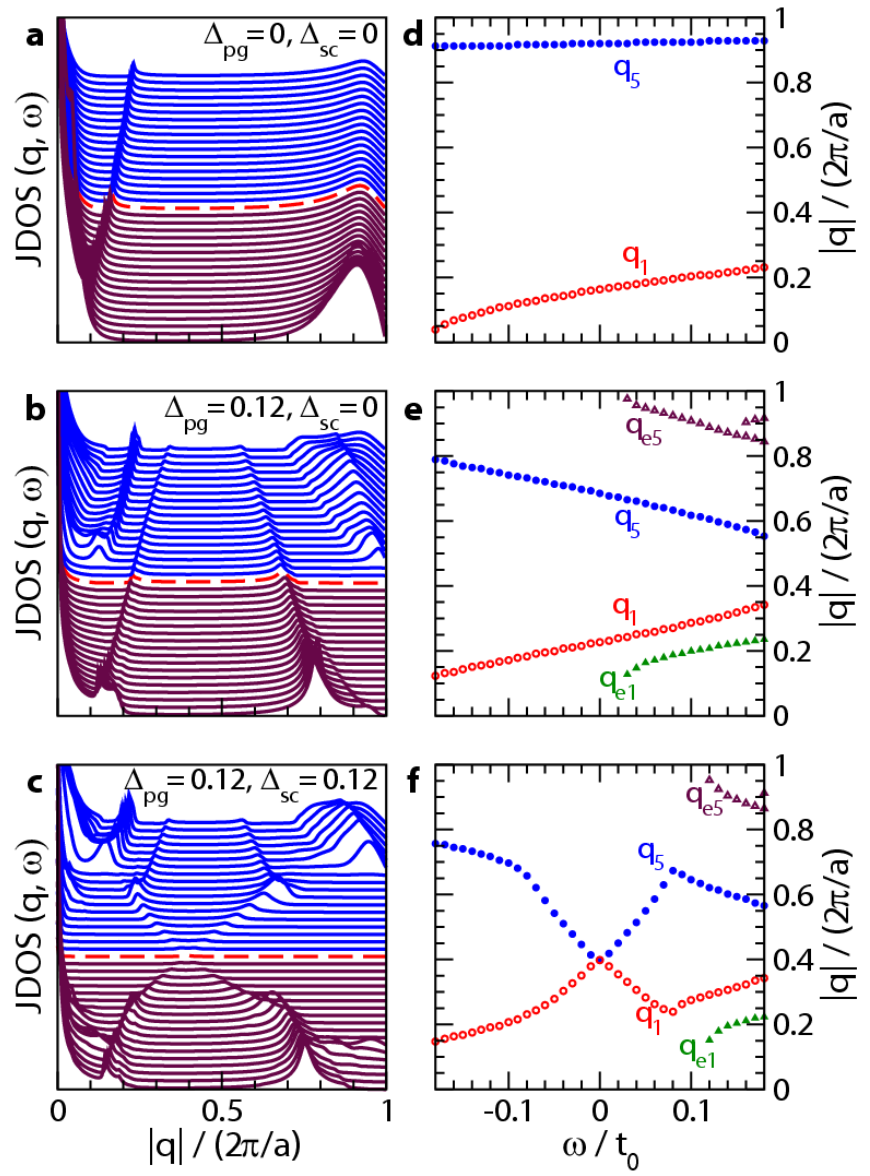

Fig. 4: (Colour online) Signatures of electron pockets in the autocorrelation function. The JDOS for $x=0.16$ in the $\boldsymbol{q}=(0,0)$ to $(2 \pi, 0)$ direction for the case of: (a) no gaps (a simple Fermi liquid), (b) $\Delta_{\mathrm{pg}}$ finite and $\Delta_{\mathrm{sc}}=0$ and (c) in the superconducting state with both gaps finite. Curves are shown offset from one another for negative bias (purple) to positive bias (blue) with steps of $\omega=0.01 t_{0}$. The dashed curve indicates the $\omega=0$ case. The variation of $\boldsymbol{q}$ versus $\omega$ following the main peaks in (a)-(c) are shown in (d)-(f), respectively. New $\boldsymbol{q}$ 's arise at positive bias indicating the existence of the electron pockets, and the Dirac point for the superconducting gap is seen in (f) at $\omega=0$. See text for further discussion.

this model to other experiments, [14, 24, 27, 33 the pseudogap Dirac point could shift to energies on order of 30-70 meV for underdoped Bi-based cuprates with $T_{c}$ of $45 \mathrm{~K}$ or less. However, as shown in frames (d)-(f), the intensity at the two Dirac points falls to zero as the density of states is vanishing at these points and so the center of the $\mathrm{X}$ feature will fall below experimental resolution. Concurrent with this may be matrix element issues which have been shown to affect the proper observation of the superconducting Dirac point. 36. Nonetheless, one can extrapolate the trend of $\boldsymbol{q}_{\mathbf{1}}$ and $\boldsymbol{q}_{\mathbf{5}}$ to find the point of intersection. While STS experiments can access both negative and positive bias to form the $\boldsymbol{q}$ versus $\omega$ dispersions, currently the positive and negative bias conductance is ratioed as $g(\boldsymbol{r},+E) / g(\boldsymbol{r},-E)$ before the Fourier transform is taken. 

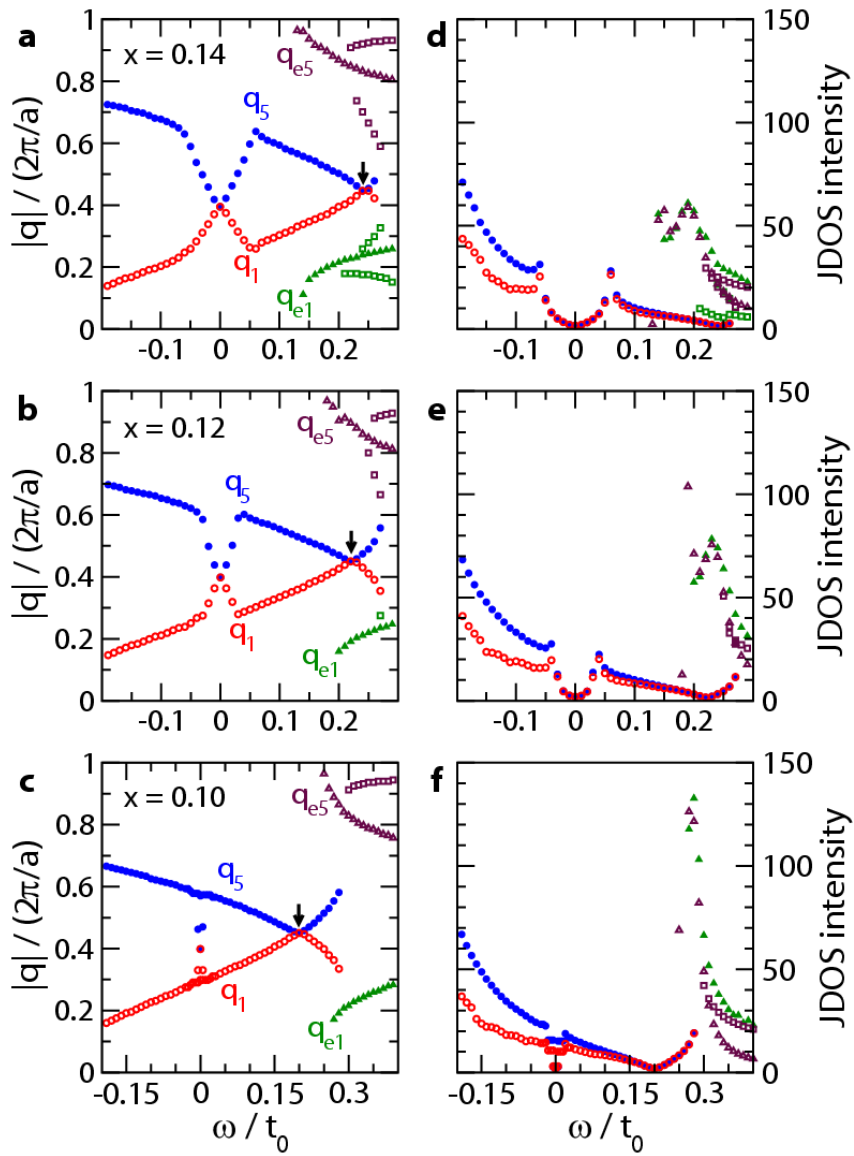

Fig. 5: (Colour online) Evolution with doping of the pseudogap Dirac point. (a)-(c) Various dopings for the underdoped phase showing $\boldsymbol{q}$ versus $\omega$ for the $(0,0)$ to $(2 \pi, 0)$ direction. In these figures, both the superconducting and pseudogap Dirac points are visible with the one for the pseudogap (marked by arrows) found at positive bias and slightly different $\boldsymbol{q}$. Extrapolation of the $\boldsymbol{q}_{1}$ and $\boldsymbol{q}_{\mathbf{5}}$ dispersions from negative bias (ignoring the $\mathrm{X}$ feature due to superconductivity) until the two intercept, should identify the pseudogap Dirac point. In the right hand frames, (d)-(f), the JDOS intensity is plotted for the $\boldsymbol{q}$ 's shown in a-c, respectively. The electron pockets provide a strong signal.

[37,38 This automatically gives the particle-hole symmetric $\boldsymbol{q}$ 's relevant for energies below the superconducting gap. To see the particle-hole asymmetry discussed here, the positive and negative biases must be kept separate. In older experimental work showing both positive and negative bias separately in a few cases, the data is given for an energy below the superconducting gap energy where particle-hole symmetry is restored [18,39]. In ref. [40], using an analysis to isolate pseudogap-only regions from an inhomogeneous gap map, a non-dispersive $\boldsymbol{q}^{*}$ is identified above the superconducting gap energy which is associated with a local "checkerboard" charge ordering. No other $\boldsymbol{q}$ 's in the pseudogap state are found although the $\boldsymbol{q}^{*}$ is not without some dispersion and is close to the $\boldsymbol{q}_{\mathbf{1}}$ value as illustrated in ref. [41]. More recently, this $\boldsymbol{q}^{*}$ feature has been measured at postive and negative bias in FTSTS for different dopings and temperatures [fig. S2(a)-(c) of ref. [42]] and has been found to disperse in the pseudogap state. This dispersion is in close agreement with the $\boldsymbol{q}_{\mathbf{1}}$ shown here for a reasonable choice of $t_{0}$. On the other hand, ARPES can only measure the negative bias. However, due to the particle-hole asymmetry, the ARPES data for $\boldsymbol{q}$ versus $\omega$ can be extrapolated to positive bias to give an estimate of the pseudogap Dirac point provided the extrapolation is taken from above the superconducting energy gap scale. Using the published AC-ARPES data presented in ref. 19 to do such an extrapolation, we note that in the one underdoped sample shown there are kinks in $\boldsymbol{q}_{\mathbf{5}}, \boldsymbol{q}_{\mathbf{7}}$ and $\boldsymbol{q}_{\mathbf{3}}$ around $22-25 \mathrm{meV}$, an indication of a possible superconducting gap energy scale. Extrapolating from energies just above this scale, we roughly estimate that $\boldsymbol{q}_{\mathbf{1}}$ and $\boldsymbol{q}_{\mathbf{5}}$ intersect at about $\omega \sim+40-55 \mathrm{meV}$ and $|\boldsymbol{q}| /(2 \pi / a) \sim 0.45-0.5$ (consistent with the results shown here). Alternatively, using $t_{0}=350 \mathrm{meV}$ in our calculations to approximately match the kink energies, we infer the Dirac point to be at $\sim 85 \mathrm{meV}$, indicating that the extrapolation from negative energy is likely to underestimate the energy of the pseudogap Dirac point. With either method, this data might be interpreted to support the presence of a pseudogap Dirac point and provides evidence for a two gap scenario. We note, however, that other AC-ARPES data on samples of higher doping and measured at higher temperature find a lack of dispersion in the pseudogap state 20,41, which they attribute to $\boldsymbol{q}$ 's connecting the tips of the energy-independent Fermi arcs, rather than charge ordering. These authors also suggest that anomalous behaviour may be seen in the dispersion of $\boldsymbol{q}$ 's in the superconducting state due to electrons coupling to collective excitations. Clearly additional study by both ARPES and STS communities could further clarify these important issues. One thing that is clear from fig. 5 is that electron pockets should provide a strong signal.

At this point, we should address further the assumption of particle-hole asymmetry which is key to our discussion. In addition to our analysis above, there is recent experimental evidence for particle-hole asymmetry in the pseudogap state from both ARPES [13,25,43] and STM experiments [14,42,44]. As we have discussed, superconductivity largely restores particle-hole symmetry at energies below the superconducting energy gap scale and hence the potential for detection of particle-hole asymmetry in experiment in the past may have been partly clouded by this issue. More recently, however, the evidence has become clearer [13] but not always made prominent 42,43. For instance, while the focus of ref. 43] was on particle-hole symmetry, they also presented evidence for asymmetry which they analyzed further in ref. 25] to find good agreement with the ansatz of Yang, Rice and Zhang 22. In fig. S2 of the supplemental information of ref. [42, particle-hole asymmetry is seen clearly in the pseudogap state, whereas particle-hole symmetry at low energies is seen in the superconducting state. There is still controversy, however, 
and hence obtaining signatures of electron pockets and the pseudogap Dirac point would help to clarify this debate.

On the issue of electron pockets, we note that the signal shown in fig. 5(d)-(f) is very large and so STS should be able to confirm the existence of these pockets. Given that they are associated with pockets in the antinodal regions of the Brillouin zone, they should not be obscured by potential matrix element effects, which are proposed to impact the nodal region. STS experiments allow us to access another part of the band structure which has been split by the pseudogap, and our results here indicate the dominance of the electron pocket signal over that of the hole pocket in this regime. This may be analogous to the quantum oscillations seen at high magnetic field in $\mathrm{YBa}_{2} \mathrm{Cu}_{3} \mathrm{O}_{6.5}$ which have been a cause for considerable debate due to the inferred presence of electron pockets 12 but a surprising lack of observation of those due to holes.

In summary, we have shown that signatures of electron pockets could be detected in FT-STS at positive bias which would confirm the reconstruction of the Fermi surface due to the formation of the pseudogap. Furthermore, AC-ARPES and FT-STS techniques can be used in a novel way to identify the pseudogap Dirac point which would both confirm a two gap scenario for the high $T_{c}$ cuprates and allow for detailed testing of candidate proposals for the pseudogap in the cuprates. Some existing ARPES data presents suggestive evidence, although somewhat indirect, that a pseudogap Dirac point is indeed present, at reasonable values of energy and momentum, and motivates further investigation particularly involving QPI FT-STS at positive bias.

\section{$* * *$}

We thank Johan Chang, J.C. Davis and J.P.F. LeBlanc for helpful discussions. This work has been supported by the Natural Sciences and Engineering Council of Canada (NSERC) and by the Canadian Institute for Advanced Research (CIFAR).

\section{REFERENCES}

[1] Timusk, T. and Statt, B., Rep. Prog. Phys., 62 (1999) 61.

[2] Le Tacon, M et al., Nature Phys., 2 (2006) 537.

[3] Boyer, M. C. et al., Nature Phys., 3 (802) .

[4] Huefner, S., Hossain, M. A., Damascelli, A. and Sawatzky, G. A., Rep. Prog. Phys., 71 (2008) 062501.

[5] Chatterjee, U. et al., Nature Phys., 6 (2010) 99.

[6] Emery, V. J. and Kivelson, S. A., Nature, 374 (1995) 434.

[7] Chakravarty, S., Laughlin, R. B., Morr, D. K. and NAYAK, C., Phys. Rev. B, 63 (2001) 094503.

[8] Zhu, J.-X., Kim, W., Ting, C. S. and Carbotte, J. P., Phys. Rev. Lett., 87 (2001) 197001.

[9] Marshall, D. S. et al., Phys. Rev. Lett., 76 (1996) 4841.

[10] Meng, J. et al., Nature, 462 (2009) 335.

[11] Yang, H.-B. et al., Phys. Rev. Lett., 107 (2010) 047003.
[12] LeBoeuf, D. et al., Nature, 450 (2007) 533.

[13] Hashimoto, M. et al., Nature Phys., 6 (2010) 414.

[14] Borne, A. J. H., Carbotte, J. P. and Nicol, E. J., Phys. Rev. B, 82 (2010) 024521.

[15] Carbotte, J. P., Schachinger, E. and Basov, D. N., Nature, 401 (1999) 354.

[16] Kirtley, J. R. et al., Nature Phys., 2 (2006) 190.

[17] Hoffman, J. E. et al., Science, 297 (2002) 1148.

[18] McElroy, K. et al., Nature, 422 (2003) 592.

[19] McElroy, K. et al., Phys. Rev. Lett., 96 (2006) 067005.

[20] Chatterjee, U. et al., Phys. Rev. Lett., 96 (2006) 107006.

[21] Wang, Q.-H. and Lee, D.-H., Phys. Rev. B, 67 (2003) 020511(R).

[22] Yang, K.-Y., Rice, T. M. and Zhang, F. C., Phys. Rev. B, 73 (2006) 174501.

[23] Borne, A. J. H., Carbotte, J. P. and Nicol, E. J., Phys. Rev. B, 82 (2010) 094523.

[24] Carbotte, J. P., Fisher, K. A. G., LeBlanc, J. P. F. and Nicol, E. J., Phys. Rev. B, 81 (2010) 014522.

[25] Yang, K.-Y., Yang, H. B., Johnson, P. D., Rice, T. M. and ZHANG, F. C., EPL, 86 (2009) 37002.

[26] LeBlanc, J. P. F., Nicol, E. J. and Carbotte, J. P., Phys. Rev. B, 80 (2009) 060505.

[27] LeBlanc, J. P. F., Carbotte, J. P. and Nicol, E. J., Phys. Rev. B, 83 (2011) 184506.

[28] LeBlanc, J. P. F., Carbotte, J. P. and Nicol, E. J., Phys. Rev. B, 81 (2010) 064504.

[29] Pound, A., Carbotte, J. P. and Nicol, E. J., Eur. Phys. J. B, 81 (2011) 69.

[30] Illes, E., Nicol, E. J. and Carbotte, J. P., Phys. Rev. $B, 79$ (2009) 100505.

[31] Bascones, E. and Valenzuela, B., Phys. Rev. B, 77 (2008) 024527.

[32] Valenzuela, B. and Bascones, E., Phys. Rev. Lett., 98 (2007) 227002.

[33] Yang, K.-Y., Huang, K., Chen, W.-Q., Rice, T. M. and Zhang, F. C., Phys. Rev. Lett., 105 (2010) 167004.

[34] Yang, K.-Y., Chen, W.-Q., Rice, T. M. and Zhang, F. C., Phys. Rev. B, 80 (2009) 174505.

[35] Khodas, M. et al., , (2010) arXiv:1007.4837.

[36] Hashimoto, M. et al., Phys. Rev. Lett., 106 (2011) 167003.

[37] Hanaguri, T. et al., Nature Phys., 3 (2007) 865.

[38] Kohsaka, Y. et al., Nature, 454 (2008) 1072.

[39] Vershinin, M. et al., Science, 303 (2004) 1995.

[40] McElroy, K. et al., Phys. Rev. Lett., 94 (2005) 197005.

[41] Chatterjee, U. et al., Phys. Rev. B, 76 (2007) 012504.

[42] Parker, C.V. et al., Nature, 468 (2010) 677.

[43] YAng, H.-B. et al., Nature, 456 (2008) 77.

[44] Pushr, A. et al., Science, 324 (2009) 1689. 